\title{
Diez años desde el Instituto Andaluz del Patrimonio Histórico
}

\author{
Román Fernández-Baca Casares \\ Director del IAPH \\ Consejería de Cultura \\ Junta de Andalucía
}

I. Foro Romano.

Asistencia al Semi-

nario italo-españo

la "Restauración

arquitectónica

hoy". De derecha a

izquierda: José

Ramón Sierra,

Alfonso Jiménez,

Bartolomé Ruiz

Fernando Villanue-

va y Román Fernández-Baca.

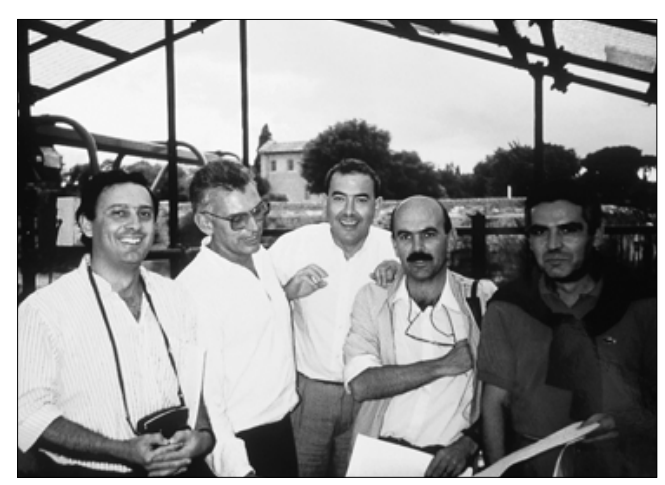

\section{La creación del IAPH}

El Instituto Andaluz del Patrimonio Histórico es una institución de la Consejería de Cultura de la Junta de Andalucía adscrita a la Dirección General de Bienes Culturales, cuyo primer acontecimiento relevante permítanme que haga un poco de historia- se produce, desde mi perspectiva relacionado con el Servicio de Conservación, y en el Seminario Ítalo-Español la "Restauración arquitectónica hoy", celebrado en la Academia Española en Roma y en San Miquele, sede del Istituto Centrale per il Restauro (ICR) y el Ministerio de Bienes Culturales, justamente en la fecha del 6 al 10 de octubre de 1987. El Seminario, que transcurrió con intensos debates y diferentes maneras de percibir la restauración arquitectónica entre italianos y españoles, estaba dirigido por Gaetano Miarelli Mariani, y contó con la presencia de Giovanni Carbonara, Sandro Benedetti, Gianfranco Spagnesi, Francisco Sisini, Salvatore Boscarino, Carlos Baztan, Manuel Manzano Monis, Fernando Villanueva, Antón Capitel, Juan López Jaén, Víctor Pérez Escolano, Antoni González, Alfonso Jiménez, José Ramón Sierra, Guillermo López Reche, Bartolomé Ruiz, entonces Director General y el que habla. (I)

Este encuentro sirvió para ver nuestras diferencias con el modelo italiano, pero también para conocer sus instituciones y organización. En concreto, visitamos el Istituto Centrale per il Restauro de la mano de Alessandro Bianchi, que hoy está aquí presente.

Prácticamente en aquellas mismas fechas culminaba el denominado Plan de Inmuebles que era un primer diagnóstico general del estado de conservación de los inmuebles de la Comunidad, sistematizando el conjunto de patologías y estableciendo la cuantía económica para su conservación. La dirección técnica encomendada a Fernando Villanueva y al Servicio de Conservación contó con la colaboración de jóvenes profesionales (José Daroca, Purificación García, Antonio Delgado, Eduardo Mosquera, Lorenzo Pérez del Campo...) y la participación en Delegaciones Provinciales del PAEMBA (Plan Especial en Materia de Bellas Artes) o selección de técnicos a través de un plan de empleo, algunos de los cuales se integraron definitivamente en la estructura orgánica de la Consejería.

El Director General y la Consejería quisieron ampliar el ámbito de programación del Plan de Inmuebles al Conjunto de la Dirección General, obteniendo con ello como resultado el bautizado Plan General de Bienes Culturales, en sentido análogo al de los instrumentos urbanísticos -Planes Generales de Ordenación Urbana- y en relación también a los del campo de la ingeniería, como son los Planes de Carreteras e Infraestructuras. Esos eran dos modelos que nos llevaban a entender los Planes Generales como excepcionales documentos de planificación y que tendrían la posibilidad de ser aplicados al campo de los Bienes Culturales.

Por tanto, en enero-febrero de 1988 culmina el Plan de Inmuebles y se inicia la realización del Plan General tras el nombramiento de Pedro Salmerón como Director Técnico. Con ello se abre la reflexión sobre la organización y los modelos referenciales (especialmente los que nos sirvieron de referencia fueron el modelo italiano de Roma, el modelo español de Madrid, y el belga de Bruselas), y comienzan los primeros intentos de Decreto de creación de la institución, donde colaboran con el Director General y el Servicio de Conservación, Guillermo López Reche, Eduardo Mosquera, José Luis Romero y Lorenzo Pérez del Campo.

La reunión Memorabilia: El futuro de la Memoria, en diciembre de 1988, durante la "Semana de los Bienes Culturales" italiana en Roma, sirvió para continuar en el conocimiento de las instalaciones y organización del ICR, ya con la idea de definir y generar un Instituto en Andalucía. En este viaje, siendo ya Director General José Guirao, se incorporan diversos profesionales que pertenecían a las Comisiones Andaluzas de Bienes Culturales (Víctor Pérez Escolano, Alfredo Morales, Ignacio Henares, Isidoro Moreno, Arturo Ruiz, Eduardo Mosquera, Lorenzo Pérez del Campo, Bartolomé Ruiz, que estaba en la Academia de Roma, y yo mismo). Más tarde se visitará el IRPA de Bruxelas, donde también asistirá Guillermo Vázquez Consuegra como arquitecto designado para rehabilitar el Área Fabril de la Cartuja como sede de la Institución. (2) (3) 
Queremos seguir profundizando en investigación, (...) impulsando la transferencia, la cooperación y los servicios especializados, continuando en la integración y territorialización, sobre todo en el marco del Patrimonio, sociedad y desarrollo.

Algo más tarde, se produce la aprobación parlamentaria del primer Plan General de Bienes Culturales, que conlleva la disposición política de crear el Instituto, la publicación del Decreto, el debate en un curso en Baeza sobre la situación patrimonial -donde también tuvimos la ocasión de ser iluminados por Alessandro Bianchi sobre el modelo de organización del ICR- y finalmente mi nombramiento como director el I de enero de 1990 por la Consejería de Cultura.

El análisis de antecedentes relativos a centros de investigación en materia de Patrimonio Histórico nos lleva inevitablemente a dedicar algunos minutos a la experiencia italiana, pionera en su época y centrada expresamente en el campo de la conservación y restauración y que si bien en este momento no es expresiva de un modelo renovador, sí representa la tradición iniciada allá por los años cuarenta y su influencia sobre nuestro presente.

En el año 1939, la inteligencia italiana de la mano de Argan y Brandi puso su interés, no ya en las ciudades históricas y el patrimonio, como ha sido una constante del pensamiento italiano de los últimos siglos, sino en la tutela del Patrimonio Histórico, como actividad de carácter fundamental.

La inercia conceptual y política de principios de siglo impulsaba el principio de prevalencia del derecho de la colectividad sobre los intereses privados en el Patrimonio Histórico, y por tanto se iniciaba la irrupción del sector público, y por tanto de la administración, en el campo operativo de los Bienes Culturales.

En otro orden la síntesis entre las nuevas aportaciones técnicas, científicas, profesionales y sociales así como la exigencia de la crítica moderna, indicaban un necesario abandono de determinadas prácticas artesanales, prefigurándose un salto cualitativo importante en el desarrollo de la actividad patrimonial.

Después de un gran número de años de trabajo, y desde mi óptica, si bien el ICR se ha constituido en una unidad muy burocrática y centralizada y con difi-

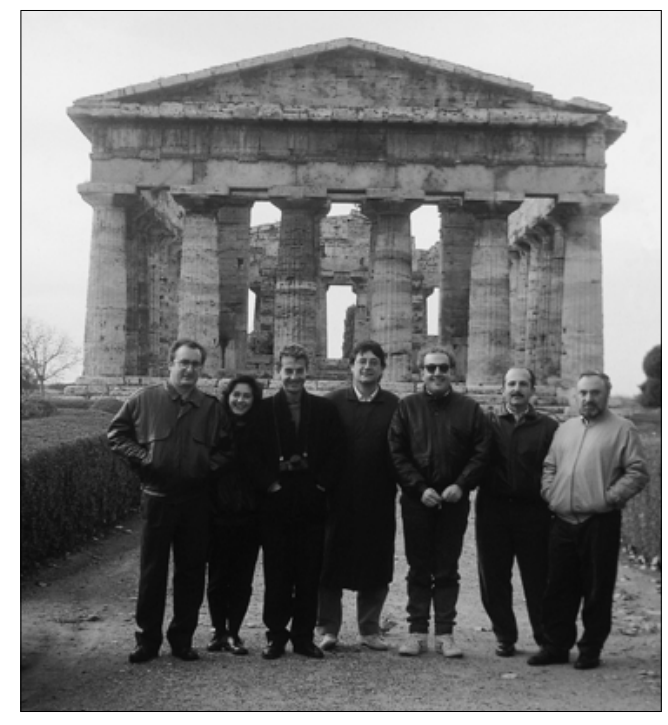

2. Visita a Paestum, de parte de la Comisión que fue al ICR en diciembre de 1988. De derecha a izquierda: Ignacio Henares, Alfredo Morales, Bartolomé Ruiz, Víctor Pérez Escolano, José Guirao y Pedro Salmerón. Fotografía de Román Fernández-Baca.

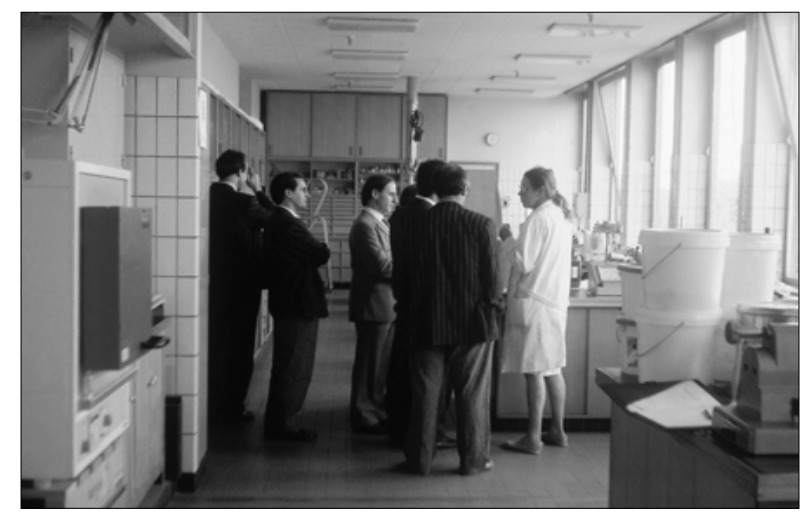

cultades para ser dinámica, también sabemos que ha

3. Visita a los talleres del IRPA de supuesto el establecimiento de una cierta tradición científica en el campo de la Conservación y Restauración de Bienes Culturales en ese país, pero con influencia internacional.

El desarrollo de proyectos y estudios de gran interés en el ámbito de la conservación, de una investigación, en materiales, alteraciones, tratamientos y formación especializada, unida a la normalización de métodos y 
técnicas, ha supuesto la extensión de las directivas del ICR al conjunto de operadores y el establecimiento del rigor científico, en el marco del proyecto crítico.

En el caso español, si bien la creación del ICROA en 1961 -veinte años más tarde que su antecesor italiano- y su posterior conversión en el ICRBC, hoy IP$H E$, se definieron a imagen y semejanza -con matices- del modelo experimental italiano, tenemos que decir que después de años de funcionamiento no existe una tradición científica trasladada mediante normalización al conjunto de operadores del país, como tampoco existe, en el panorama conceptual y metodológico español, una transferencia sistemática de su experiencia. Cabría destacar, sin embargo, el esfuerzo individualizado de profesionales del IPHE, que, como conjunto interdisciplinar, no ha sido suficientemente aprovechado.

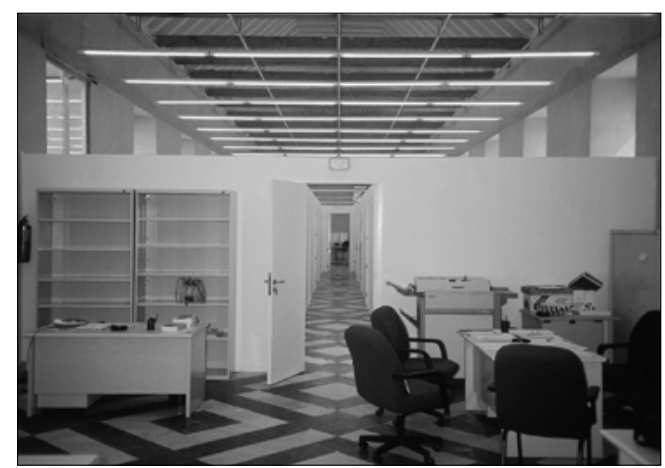

Su primera estructura, que es la estructura que conocíamos -esta mañana hemos visto otra estructura muy reciente- se inicia con un área de estudios previos, departamentos de información e investigación, áreas de bienes muebles y bienes inmuebles y área de programas. Una estructura, en definitiva, que se sitúa muy en el campo del objeto, donde la documentación, también objetual, no parte de la aplicación de técnicas documentales, gráficas y su sistematización y donde la formación, transferencia y normalización de trabajos no cobran un alto grado de desarrollo, según nuestro criterio.

Con este panorama, nuestra andadura se inicia, como decíamos, en el marco del Plan General de Bienes Culturales, con una primera observación: la creación del Instituto Andaluz del Patrimonio Histórico se produce en este marco regional planificado de los bienes culturales. Por lo tanto su creación no es discrecional, sino a través de un programa especial, en el marco de una organización territorial, donde al Instituto se le definen con claridad sus funciones como Institución especializada. Sirva de comentario que el mencionado Plan General desarrolla actividades bastante novedosas en el campo del patrimonio, tiene vocación integradora -aunque con el tiempo, quizás, no la alcanzara plenamente- y está apoyado en un estudio económico-financiero. Ahora que tanto hablamos de patrimonio y desarrollo, realmente podría ser un ejemplo de lo que puede ser un instrumento de esta naturaleza, incipiente evidentemente en aquellos tiempos.
En este marco, el Instituto Andaluz del Patrimonio, como en las grandes empresas, sería una unidad especializada, que no busca reproducirse en el territorio, sino que demanda un papel singular, fundamentalmente en el campo de la investigación y los desarrollos, y hoy día también en la innovación, para dar asistencia especializada, para trabajar en proyectos modélicos y para transferir sus trabajos y conocimientos mediante servicios especializados. Sus funciones están básicamente contenidas en su Decreto y son sintéticamente las de realizar estudios científicos, el desarrollo de teorías, métodos y técnicas, la documentación e información, la investigación de la tutela y sus instituciones, la ejecución de programas, planes y proyectos, la formación especializada, así como la colaboración y cooperación en el marco de sus actividades.

En su estructura general, especificada en el Decreto -como muy bien Miguel Ángel Castillo ayer comentaba- se definían tres áreas: documentación, intervención y Secretaría, sin presencia de la formación y de la comunicación, que, sin embargo, fueron rediseñadas en el tiempo. De modo que la estructura actualizada se sustenta sobre cinco áreas: documentación, intervención, formación, comunicación y, de reciente incorporación, arqueología subacuática.

Tras una primera estancia física en lo que fue el Archivo General de Andalucía en aquellos primeros años 90 , hace ya más de 10 años, se constituye el primer grupo de profesionales, o equipo fundacional del Instituto: José María del Pozo, Eugenio Fernández, Eduardo Mosquera, José Daroca, Purificación García, Juan Jesús Jiménez, María José González, Raniero Baglioni, María del Carmen Ladrón de Guevara, Angeles Gil, Rosario Villegas, Isabel Ortega, Valle Muñoz, Marcelo Martín, Carlos Romero, Gabriel Ferreras, María Luisa Marín, Luis Martínez Montiel, Elisa Torrente... a los que se ha ido añadiendo un largo etcétera, que se ubica en unas oficinas provisionales en la misma Cartuja de Sevilla (4).

Las obras del Instituto dirigidas por el Estudio Vázquez Consuegra en el Área Fabril de la Cartuja, tras la elaboración del programa de usos por nosotros y los estudios previos del equipo de rehabilitación de la Cartuja, fue un proyecto seleccionado para la Bienal de Arquitectura años más tarde. Desde el proyecto de Guillermo Vázquez Consuegra se consigue una difícil armonización entre construcciones preexistentes y nueva arquitectura, entre espacios donde estructura y construcción, con hormigón, hierro y vidrio, conectan con las figuraciones de las arquitecturas industriales de Pickman. En suma, un proyecto que, a pesar de su contemporaneidad adquiere gran consenso cultural y profesional, teniendo una interesante contradicción: si bien el empleo del hormigón, vidrio e hierro genera cierto contraste con los materiales preexistentes y por tanto hablamos "de contrastación" en la materialidad de la obra, el conjunto espacial y arquitectónico es ciertamente analógico, por la similitud entre las nuevas construcciones operadas-transformadas y las preexistencias industriales. (5) 


\section{Primeros Documentos y Acciones}

Los primeros documentos y acciones que realiza el Instituto Andaluz del Patrimonio en estos años constitutivos estuvieron impregnados de ciertas dificultades. Desde el empleo de la planificación anual se producen diversos documentos, de los que destacaría el documento elaborado el año 1994 denominado Concepto, funciones y servicios del Instituto Andaluz del Patrimonio. Función social y propuesta de planificación, cuyo contenido, más de carácter teórico, ya nos hablaba de algunos fundamentos consolidados posteriormente, como la armonización de criterios y necesidades técnicas con la rentabilidad social, la suma de conocimientos o acción interdisciplinar en todos los proyectos del Instituto, la búsqueda de un lenguaje común y por tanto de una componente transversal de corte generalista, a la vez que un mayor nivel de especialización en la búsqueda de nuevos perfiles en relación al Patrimonio, la acción patrimonial donde conviven generalidad y especialidad... También decíamos que esta tarea es de toda la organización, no sólo del Instituto, y es importante resaltar esta apreciación, ya que se tiende a justificar al IAPH en este papel y pensar que otras instancias no tienen esta responsabilidad, aunque, en este marco, no obstante, a la Institución le corresponde el impulso y desarrollo de trabajos más especializados, vértices de la acción genérica patrimonial, apertura de fronteras, caminos, o allí donde exista un mayor nivel de dificultad o empleo de tecnología.

El documento profundiza en las diferentes áreas del Instituto. Este es el caso del Centro de Documentación, que busca su sentido en el ofrecimiento de información a los administradores, profesionales, investigadores y ciudadanos en general, permitiendo la consulta ágil, el acercamiento del patrimonio a la sociedad, además de ser un ejercicio de transparencia e instrumento para la cooperación. Todo ello en el marco de un sistema en Red donde el Centro de Documentación ocuparía una posición preeminente y donde las líneas fundamentales se desarrollarían a través del desarrollo de la documentación, mediante bases de datos y la generación de los servicios de información públicos.

El Centro de Intervención, antes del documento del año 94, inició el proyecto y actuación de Acondicionamiento Museístico y Conservación de las colecciones de la Capilla Real. Este proyecto y actuación fue determinante. Nuevas formas de entender la conservación vinieron de la cooperación con el ICR y el IRPA de Bruselas y Pedro Salmerón, que nos enseñaron nuevas maneras de abordar estas actuaciones, impregnadas de un rigor metodológico y un gran cuidado -sensibilidad-que, entiendo, se han mantenido en la institución. Este proyecto tan difícil, permitió aproximarnos a conocer las técnicas y métodos de análisis -tan importantes para montar nuestros laboratorios-, manejar una interpretación histórico-cultural más apoyada en las ciencias y técnicas aplicadas, profundizar en la museología, nuevas técnicas museográficas, relación del objeto con el contexto o conservación preventiva, etc. y se iniciaron algunas for-

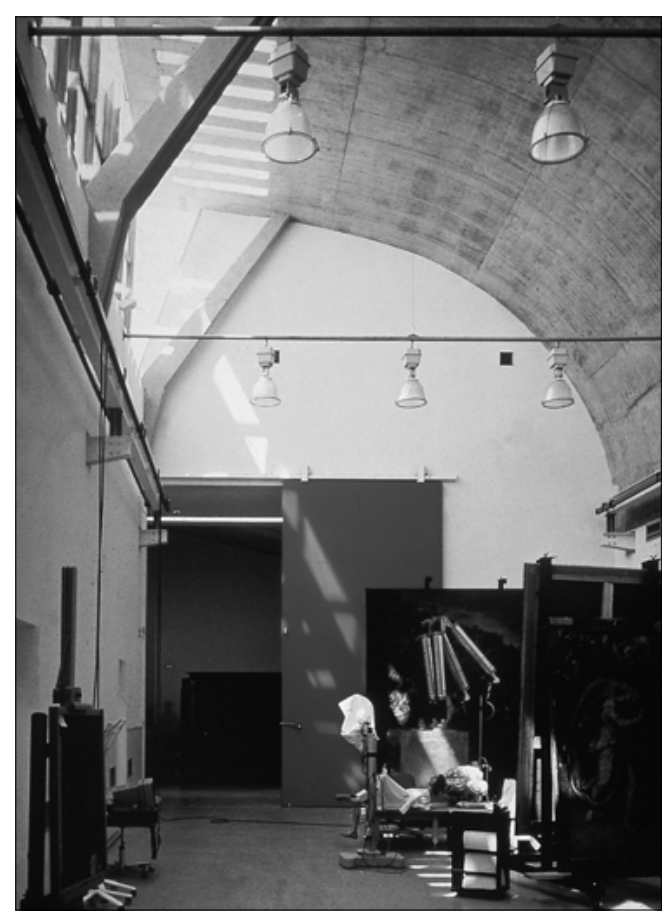

5. Rehabilitación de la Cartuja. Área Fabril. Arquitecto Guillermo Vázquez Consuegra. maciones profesionales muy especializadas que han nutrido nuestros talleres. Pedro Salmerón, María José González, Raniero Baglioni, Carlos Cacace, Alessandro Bianchi fueron algunos de los profesionales que dirigieron y coordinaron el proyecto.

En el documento de 1994, el Centro de Intervención alude al perfeccionamiento de los criterios y métodos de conservación-restauración, en un panorama donde existía la persistencia de tratamientos incompatibles sobre los Bienes Culturales, de desarrollo de instalaciones costosas, no suficientemente adecuadas para las colecciones, de falta de rigor y estudios previos en el campo de la conservación... Se propone la intervención como investigación técnica, metodológica, de gran contenido experimental, interdisciplinar y didáctica, el establecimiento de acciones de conservación preventiva, de normalización y control de calidad en bienes culturales, dando servicios especializados mediante asistencia técnica, laboratorios y talleres de restauración.

En el Área de Formación, tras consultar con cuarenta profesionales de los bienes culturales el Programa de Formación y alcanzar un consenso bastante amplio en relación a sus contenidos, se fue decantando una formación en tres ámbitos específicos, que serían matizados más tarde. Estos tres ámbitos corresponderían al ámbito de formación profesional, del personal propio - de la Administración y de las Instituciones-, y becas de formación e investigación que con el tiempo han ido también matizándose y ajustándose por el propio departamento.

En el Área de Difusión o Comunicación tendríamos que contar las dificultades que tuvimos para poner en marcha el Boletín PH. Este se inició con unas primeras hojas que permitieron desarrollar una puesta en marcha de la publicación, enriquecida con la colaboración 


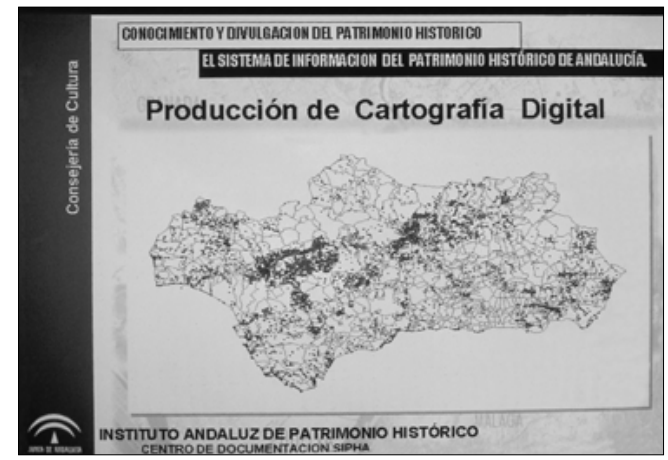

e ilusión de todos. Posteriormente otras líneas, como son los Cuadernos y Cuadernos Técnicos, eran el resultado de un número importante de encuentros, debates, jornadas, que fomentaban la confrontación de criterios, el conocimiento de actuaciones y la transferencia, estableciendo un marco de conexión del IAPH con el mundo profesional y cultural.

\section{La gestión de estos diez años}

En el año 1996, siendo Director General de Bienes Culturales Lorenzo Pérez del Campo, Consejero de Cultura José María Martín, y Viceconsejero Bartolomé Ruiz, se duplica el presupuesto del Instituto Andaluz del Patrimonio Histórico, pasando de 170 a 340 millones de pesetas. En 1999 se incrementa hasta 479 millones de pesetas, siendo Director General Marcelino Sánchez, Consejera de Cultura Carmen Calvo, y Viceconsejera Claudia Zafra.

El Instituto ha desarrollado lo que consideramos una primera etapa en estos primeros diez años. El trabajo del Centro de Documentación, considero que conocido ya en todo nuestro país e incluso empieza a conocerse más allá de nuestras fronteras, canaliza su actuación según tres objetivos básicos, coherente con su estructura: desarrollo y producción de la información del patrimonio, aplicación de técnicas gráficas e informáticas (segundo departamento de carácter más instrumental) y servicios de información a los usuarios. El Centro, como él mismo nos dice, tiene el encargo en el II Plan General de Bienes Culturales de desarrollar el Sistema de Información del Patrimonio Histórico de Andalucía o SIPHA, definido como conjunto integrado de información relativa a los elementos de dicho patrimonio y al ambiente $y$ territorio en el que están inmersos, basado en principios de integración y coordinación de las diferentes Instituciones y Organismos que contienen documentación e información sobre el patrimonio en nuestra región.

El Centro parte del principio del análisis territorial en el que los bienes culturales están insertos más allá del objeto y de los valores estéticos, históricos y funcionales, que también son considerados, incorporando nuevas tecnologías y métodos analíticos encaminados a la comprensión global del patrimonio, trabajando a diferentes escalas.
El SIPHA es un proyecto de conocimiento global del Patrimonio en el marco regional y sus territorios. Contiene información relativa a los Conjuntos Históricos y bienes arquitectónicos, arqueológicos, muebles y etnológicos. Incorpora un lenguaje e imagen normalizado y las fuentes documentales y bibliográficas. Establece entre otras informaciones la situación jurídica, el estado de conservación, y un vínculo entre bienes relacionándolos. Actualmente se desarrolla la georreferenciación e integración de los bienes en los territorios de Andalucía, ya culminados en algunos casos, como corresponde al Patrimonio Arqueológico. (6)

El volumen total de información asciende a 176.000 registros patrimoniales a los que puede accederse mediante los Servicios de Información.

Bien a través de los Servicios puntuales de información, para dar respuesta a peticiones concretas de información, bien a través de los Servicios de Información del Fondo Bibliográfico-Gráfico o finalmente utilizando Internet donde queda recogido un compendio de lo que es el Instituto, sus actuaciones más relevantes, sus cursos, boletines y solicitudes de información.

Las actuaciones del Centro de Intervención tienen como objetivo el conocimiento y la investigación para la intervención, la actuación propiamente dicha y su transferencia. Trabaja desde el proyecto de intervención, como instrumento, constituido según tres actos metodológicos. El primero, centrado en el conocimiento de los valores y significación cultural del objeto, así como su diagnóstico y evaluación de tratamiento -entendemos que en un mismo objeto pueden superponerse valores de distinta índole como pueden ser valores sociales, históricos, artísticos, antropológicos, etc.-, el segundo acto, en clave proyectual de conservación y recuperación de los valores de uso y expresivos de la obra, y el tercer acto, de transferencia profesional y social implementada en estrategias más amplias de desarrollo cultural y formación profesional.

El primer acto de conocimiento está encaminado a la comprensión desde diversas perspectivas relacionadas con el examen directo y técnico de la obra y entendido en toda su extensión, no exclusivamente en la evaluación de los valores históricos arqueológicos sino también en la valoración de otros valores expresivos, espaciales, constructivos, inmateriales, ambientales, etc., actividades que han tenido que ser puestas a punto durante estos últimos seis años, y en las que han sido fundamentales las tareas de los laboratorios del Instituto y otras del mismo Centro. Técnicas que han sido desarrolladas para el conocimiento material de los bienes, las alteraciones que inciden sobre los mismos, el análisis del medio ambiente en el que está inmerso...

El desarrollo de estas técnicas no ha sido puesto a punto exclusivamente para llevar a cabo acciones concretas, sino también como servicio especializado a disposición de la organización general de los Bie- 
nes Culturales y de especial utilidad para las Instituciones del Patrimonio, ya que los servicios para la intervención están en permanente progreso y su aplicación puede suponer un apoyo importante donde recurrir en un marco de relaciones.

Estas técnicas también nos han permitido trabajar, no sólo en el ámbito del proyecto operativo y directo sobre el Bien Cultural, sino en acciones o recomendaciones de carácter preventivo aplicado a un sinfín de bienes, teniendo como paradigma la actuación de la Capilla Real de Granada.

La relación de proyectos del Centro de Intervención es quizás muy extensa, con una media de cincuenta proyectos al año, de gran dificultad y componente investigadora, existiendo poca actividad en el campo de bienes inmuebles. Esta actividad ha venido acompañada de la consolidación de los talleres de pintura, escultura, tejidos, papel, material arqueológico y metales, y los laboratorios de métodos de examen físico, composición de química, alteración biológica, paleobiología y materiales de construcción. (7)

Actuaciones como La Inmaculada y La Santa Cena de Murillo, Santa Catalina y Santa Bárbara de Esturmio o La Derrota de los Sarracenos de Valdés Leal representan algunas de las actuaciones significativas en Pintura.

El Retablo Mayor de la Capilla Real de Granada, el Señor de Pasión de Martínez Montañés, la Virgen de la Amargura, el Cristo de Burgos o de la Salud de San Bernardo, la Virgen de la Victoria patrona de Málaga son algunas referencias del lugar tan destacado que ha cubierto el Centro de Intervención en el campo escultórico, cuya misión ha sido también explicar a través de múltiples ejemplos cómo actuar sobre Escultura Policromada con rigor y considerando los valores inmateriales que contienen estos bienes.

La actuación en el Pendón de la ciudad de Sevilla en Tejidos, el Hypnos de Almedinilla en metales o el inicio de las tareas de investigación de material Arqueológico y Metales pueden ser otros referentes indudables sobre los que se ha trabajado y se incide en la actualidad. (8)

En el ámbito de inmuebles, además de numerosos informes y actuaciones ya relatados como es la Capilla Real de Granada, podemos referir algunas actuaciones, en colaboración, como el proyecto integral para la Santa Cueva de Cádiz o el proyecto realizado para la muralla nazarí de la Plaza de la Marina de Málaga, donde el reflejo del vidrio crea una ilusión que multiplica la muralla o, finalmente, actuaciones como la realizada en la Puerta de Córdoba, que ha venido precedida de una detallada investigación arqueológica, clave para la restauración culminada recientemente. (9)

El Departamento de Formación es un área del Instituto que ha ido con el tiempo ocupando un espacio más amplio y dinámico y siempre desde el consenso en su programa. Tiene como máxima, que compar- te con otras áreas del Instituto, la definición del Patrimonio como unidad de acción donde confluyen diferentes disciplinas académicas, colectivos profesionales, instituciones, asociaciones y organizaciones ciudadanas, con el fin de favorecer el desarrollo social y perpetuar la memoria para el futuro. Formación, pues, que considera la visión integral y territorial de los bienes culturales entendidos como recurso en sus diferentes formulaciones y profundizando en los instrumentos de interrelación con la protección, conservación y el acrecentamiento en el patrimonio. El departamento ha desarrollado sus actividades de Formación inicial, mediante diferentes masters en colaboración con las Universidades andaluzas, de Formación de especialización, en colaboración con múltiples asociaciones y Universidades (quiero aludir a que prácticamente en este año han sido veinte las asociaciones que han colaborado con el área de formación), y de Formación personalizada, mediante el programa de becas del Instituto.

Un análisis cuantitativo del propio departamento nos muestra el gran esfuerzo que hace. En el año 1999 se han realizado 31 cursos en el territorio de Andalucía, 4 masters, y se ha formado a unos 900

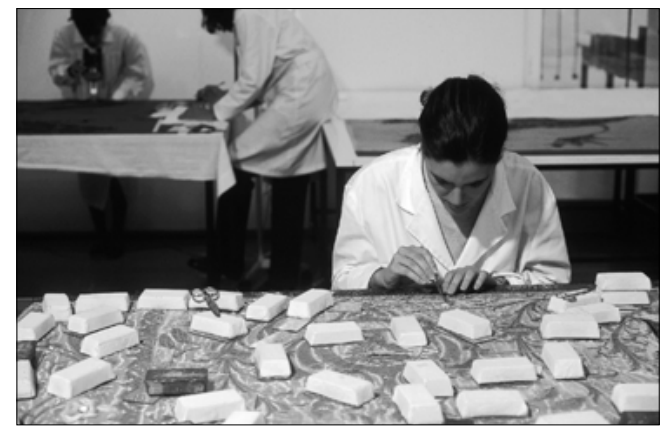

7. Trabajos en el Taller de Tejidos del IAPH.

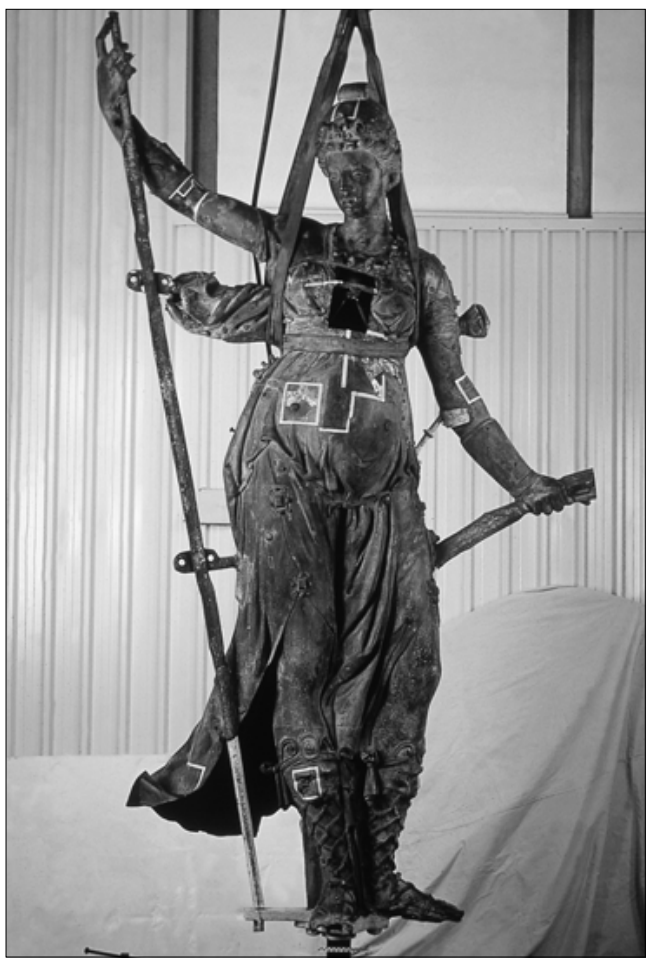

8. Trabajos en el Giraldillo de Sevilla. 

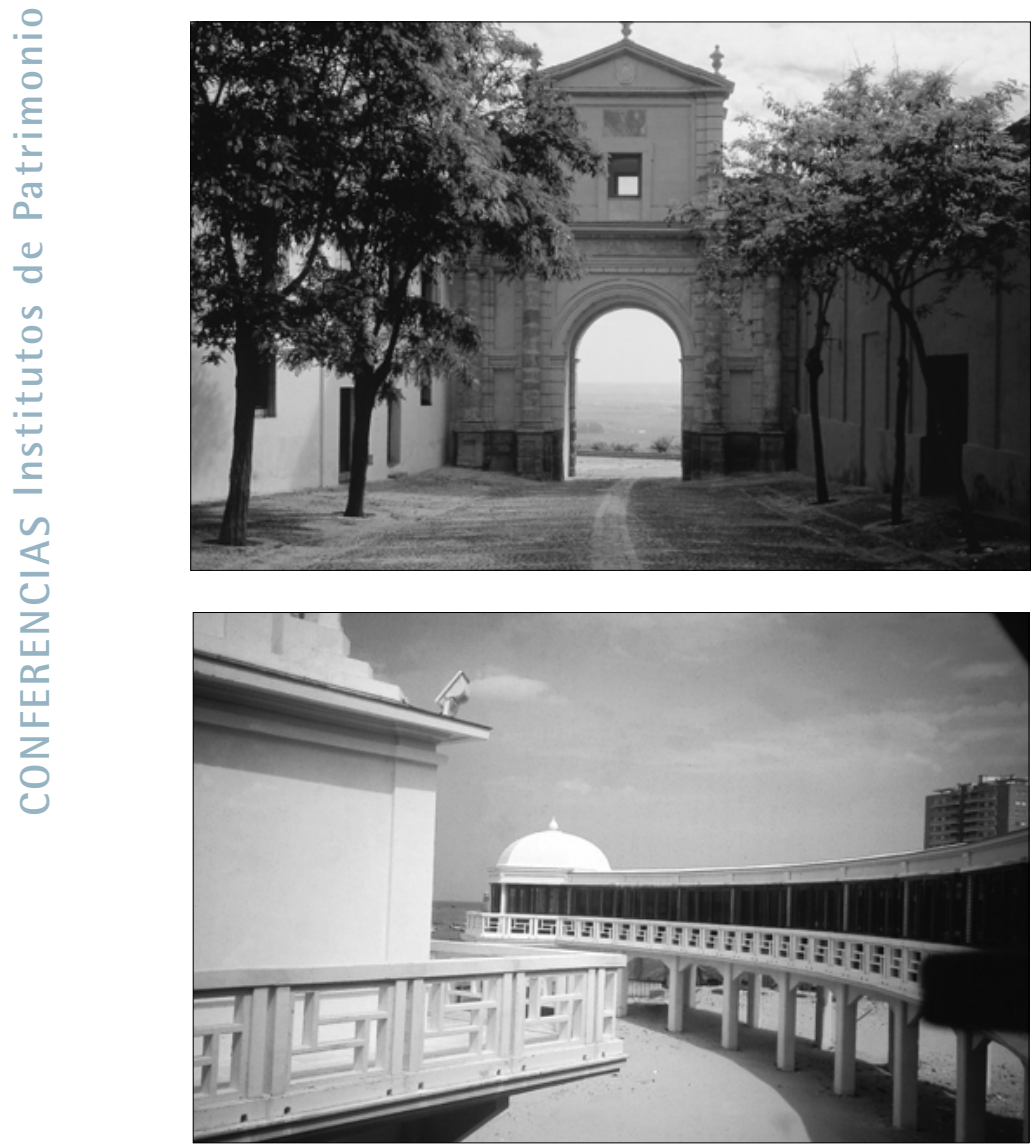

9. Restauración de la Puerta de Córdoba.

10. Balneario de la Palma de Cádiz, sede del Centro de Arqueología Subacuática. alumnos, todo ello con sólo cuatro profesionales en el área y reducidas fuentes financieras. Yo creo que con muy pocos recursos humanos y financieros se ha desarrollado una tarea ingente.

El Departamento de Comunicación es también otro grupo reducido y multifuncional de profesionales. Desde la necesidad avalada, tanto desde el Decreto de creación del propio Instituto como por el principio de comunicar e informar (principio que corresponde a distintos estamentos de la Administración Pública), se produce este esfuerzo del departamento, también con reducidos medios, por optimizar sus servicios a la vez que lograr la aprobación y el reconocimiento público de los mismos. El marco de este programa viene dado por el papel mediador en torno al Patrimonio, dentro del cual la difusión, actualización profesional y debate interdisciplinar son objetivos prioritarios.

Las estrategias de trabajo se desarrollan en dos niveles: la difusión del trabajo generado por el propio Instituto y la coordinación y administración de recursos dispersos dirigidos a la difusión del Patrimonio creados por otros organismos públicos y privados.

Son instrumentos sistemáticos de comunicación su propia línea editorial, que es la línea editorial creada por el departamento a través de PH Boletín -esta mañana aludía alguien a la importancia de esta revista, como revista profesional e independiente, y ciertamente intenta ser independiente y servir de transferencia y debate interdisciplinar con treinta números editados hasta hoy. También Cuadernos y Cuadernos
Técnicos como colecciones han incorporado el resultado de debates, encuentros y jornadas sobre criterios y metodologías en un esfuerzo de transferencia profesional. En el campo de las exposiciones, de las visitas y de las jornadas, el departamento ha realizado algunas exposiciones de las cuales, por su importancia, destacaría la presentada en Évora, en el marco del Encuentro entre las Ciudades Patrimonio de la Humanidad.

Finalmente el Centro de Arqueología Subacuática de nueva creación y en constitución tras la rehabilitación, no sin problemas, nace para investigar, documentar, intervenir y conservar el patrimonio arqueológico subacuático. En este período se trabaja en el inicio de las tareas documentales del patrimonio arqueológico subacuático y la creación de la unidad de información del CAS, en la generación de cartas de riesgo como instrumento decisivo para la protección del mismo, además de combatir el expolio. Entre sus primeros establecimientos se encuentran la constitución de los laboratorios, el taller general de conservación y el desarrollo de proyectos de investigación tendentes a la generación de medidas preventivas de conservación, protección, así como la sensibilización sobre el Patrimonio arqueológico subacuático. (10)

\section{El Patrimonio hoy y el modelo de Centro}

La concepción genérica del patrimonio histórico tiende a descomponerse en patrimonios sectoriales, no sólo por tener reconocimiento normativo desde la ley de Patrimonio Histórico de 1985, sino porque cada uno de ellos ha logrado una presencia social y también un importante desarrollo científico y disciplinar. Es el caso de los patrimonios moderno, industrial, subacuático, inmaterial, etc. que por diversas razones buscan un lugar y una presencia efectiva frente a otros patrimonios más tradicionales.

Si a esta ampliación conceptual del patrimonio añadimos también que cada bien cultural es diferente y distinto en sus partes y que se halla en un medio físico determinado, con afecciones de distinto signo, podemos darnos cuenta de la gran complejidad que supone hoy día el tratamiento y la acción en bienes culturales.

El patrimonio histórico, como un recurso cada día importante, adquiere una presencia y complejidad del mismo orden que otros recursos fundamentales. En este sentido, se han pronunciado los integrantes del Foro de Andalucía en el Nuevo Siglo en la introducción de Andalucía Cohesionada:

"La utilización que se haga de los recursos del territorio, la disposición que tengan en él los usos o aprovechamientos, la localización de las actividades y los asentamientos de población, la implantación y desarrollo de las principales estructuras y sistemas territoriales, intervienen decisivamente en el desarrollo, bienestar de los andaluces y condicionan las posibilidades de las generaciones futuras. 


\begin{abstract}
El territorio, definido en su forma más amplia, es también naturaleza, reserva de recursos y ecosistemas. (...) Es necesario un nuevo pacto social y natural basado en el conocimiento del medio fisico y en una actuación cuidadosa, no sólo para aprovecharlo mejor o para evitar riesgos y peligros reales, sino también por respeto a sí mismos, como componentes y primeros integrantes de la naturaleza".
\end{abstract}

Estos párrafos señalan los problemas y soluciones del patrimonio histórico. Si bien asistimos a la persistencia de los riesgos e impactos de la época postindustrial, causantes de un gran deterioro general, como la aceleración de los procesos de destrucción, del empobrecimiento de los entornos, de la agresión a las ciudades y al medio ambiente, éstos se intentan minorar con el régimen de protección del patrimonio que:

- Exige un cambio de orientación en los comportamientos, en la manera de conocer, intervenir e investigar, en la armonización de conservación y desarrollo, en la generación de una economía sólo posible si se producen modelos más favorables.

- Obliga a relacionar bien cultural, natural e inmaterial sobre un mismo territorio y dentro de un sistema complejo que requiere un gran esfuerzo de suma de conocimientos y disciplinas que el Sistema de Información del Patrimonio Histórico de Andalucía (SIPHA), creado dentro del Programa de Documentación del Instituto, tiene la vocación de integrar.

- Genera un gran esfuerzo investigador, operativo, formativo, educacional que hace uso de los recursos tecnológicos, de los conocimientos de disciplinas básicas y aplicadas del patrimonio histórico y de la competencia profesional y política necesaria para actuar.

- Hace necesaria la articulación de organizaciones preparadas para abordar estos retos del siglo XXI en las misiones globales de tutela sobre el territorio regional y para la acción concreta experimental.

- Significa que la denominación y organización del Instituto como de Patrimonio Histórico es más adecuada que la de Conservación, ya que atiende al conjunto de actividades asociadas al hecho patrimonial y no exclusivamente a las relativas a conservación.

Por tanto, esto, a todos, especialmente también a nosotros, nos obliga a un gran esfuerzo investigador, operativo, formativo, educacional, que requiere el uso de la imaginación, de la creatividad, de recursos tecnológicos, de la suma de conocimientos y alianzas en el proyecto patrimonial, de la articulación de organizaciones preparadas para abordar estos retos del siglo XXI en las misiones globales de la tutela, eje de la acción, y también en los desarrollos concretos experimentales y en los desarrollos en los territorios periféricos de Andalucía.
Nosotros, desde este Instituto, queremos estar situados en el marco de la innovación, atentos a la función social de nuestra actividad, mediadores entre agentes e Instituciones en el Patrimonio, permanecer como unidades dinámicas impulsoras de la dinamización, y estar vinculadas al sistema de trabajo de la organización. También como Institución definidora de criterios, normalización, metodología, nuevas tecnologías, proyectos y producción de calidad en el Patrimonio, sin menospreciar la creatividad, y avanzando en la sensibilización en el Patrimonio según tres ámbitos: una unidad de conocimiento global, una unidad de intervención, y una unidad de transferencia, que corresponden hoy la primera al Centro de Documentación, la segunda al de Intervención, y la tercera al ámbito de Formación-Comunicación.

Necesitamos consolidar la Institución definitivamente. Es débil en su estructura administrativa y en sus recursos humanos. Debemos reforzar lo que es la transferencia con otras unidades de la propia organización y externas a la propia Administración, que genere una mayor coordinación inter-administrativa de carácter transversal y optimización de los recursos administrativos. Queremos seguir profundizando en investigación, considerando esos otros marcos que ya son de referencia, como es el marco andaluz, el marco nacional y europeo de investigación, desarrollando un ajuste de la organización en el marco de una regulación interna que nos permita proyectos más transversales, impulsando la transferencia, la cooperación y los servicios especializados, continuando en la integración y territorialización, sobre todo en el marco del Patrimonio, sociedad y desarrollo, y apostando por un intento de mayor sensibilización hacia las nuevas generaciones en el campo del patrimonio. (I I)

Llegados al final de esta ponencia, quisiera agradecer la ayuda de tanta gente. Políticos que nos han permitido avanzar en nuestra trayectoria. Profesionales y equipo del Instituto que ha desarrollado con su trabajo y vocación lo que hoy somos entre todos. También a los amigos de la Dirección General y Delegaciones y a tantísimos colaboradores que han contribuido también a desarrollar nuestro proyecto I+D. A todos muchos gracias.

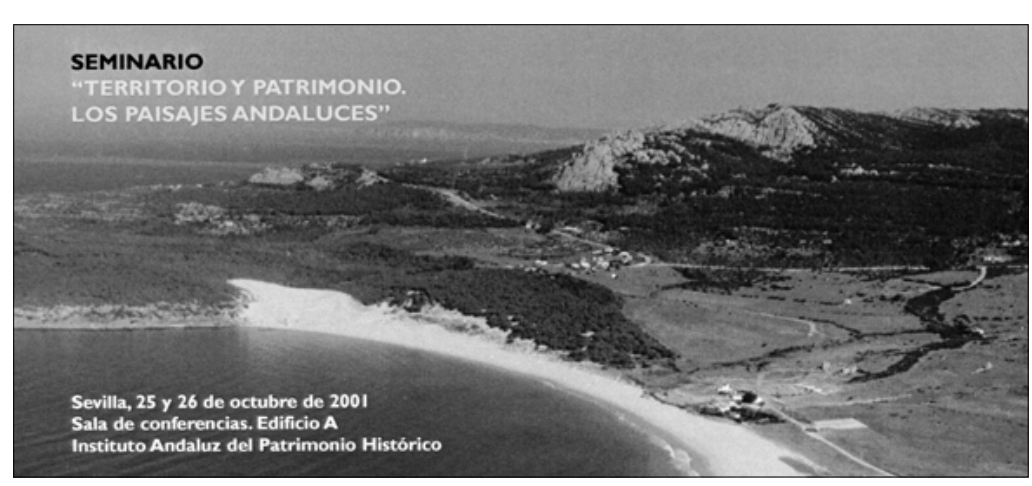

